\title{
An improved Metropolis algorithm for hard core systems
}

\author{
A. Jaster \\ Universität - GH Siegen, D-57068 Siegen, Germany
}

\begin{abstract}
We present an improved Metropolis algorithm for arbitrary hard core systems in any dimensions. In the new updating scheme the conventional Metropolis step of a single particle is replaced by a collective step of a chain of particles. For the two-dimensional hard sphere model we show that this algorithm essentially reduces autocorrelation times in the vicinity of the transition point.
\end{abstract}

\section{Introduction}

The two-dimensional melting transition of the hard sphere system has been an unsolved problem for many years ${ }^{1}$. There are several theoretical approaches for the description $[2,3]$ and many numerical investigations have been done to analyze kind and order of this transition. The order-disorder transition was first seen in a computer study by Alder and Wainwright [4]. They performed a numerical simulation with the molecular dynamics algorithm (constant $N V E$ simulations). Recent Monte Carlo investigations were done in the NVT [5,6] and $N p T$ ensemble $[7,8]$. Unfortunately, these simulations gave non-unique results.

One of the main problems of Monte Carlo simulations is the autocorrelation in the sequence of generated configurations. This leads to a drastical increase of computer time when the system is in vicinity of the phase transition point. Roughly speaking, the autocorrelation time scales like $\tau \sim \xi^{z}$, where $\xi$ is the correlation length and $z$ denotes the dynamical critical exponent. This phenomenon is called critical slowing down. In particular, conventional local algorithms such as the Metropolis algorithm are affected by this problem $(z \approx 2)$. For some simple spin systems, a dramatic reduction of critical slowing down

$\overline{1}$ A review of two-dimensional melting is given in [1]. 
$(z \approx 0)$ can be achieved by the cluster algorithm $[9,10]$. An attempt to implement such an algorithm for hard core systems was proposed [11] ${ }^{2}$, but several complications arose near the critical point. Also, no practical investigations of autocorrelation times were made.

This paper presents an improved Metropolis algorithm, applicable to arbitrary hard core systems in any dimensions. For that purpose the usual local Metropolis algorithm [13] is replaced by a non-local chain Metropolis algorithm. In the special case of hard disks in two dimensions we show that this leads to a remarkable reduction of autocorrelation times.

\section{The algorithm}

Normally a Metropolis step for an $n$-dimensional hard core system consists of the following operations:

(1) Select a particle $i$ from the system (this can be done randomly with equal probability or by sweeping over the whole system).

(2) Displace the particle from its position $\vec{r}_{i}$ with a uniform probability (i.e. $\sim d V)$ to any point inside a cube ${ }^{3}$ of size $(2 \epsilon)^{n}$, centered at $\vec{r}_{i}$.

(3) Accept the step, if the hard cores do not overlap.

The idea of the improved Metropolis algorithm is to displace chains of particles, thus enhancing the efficiency. For simplicity and to decrease random number generations, all particles of a chain are moved in the same direction with equal displacements. Thus the improved (chain) Metropolis step is as follows:

(1) Select a particle $i$ randomly with equal probability from the system.

(2) Select with uniform probability a vector $\delta \vec{r}$ of a cube of size $(2 \epsilon)^{n}$, centered at the origin.

(3) Displace the particle from its position: $\vec{r}_{i} \rightarrow \vec{r}_{i}{ }^{\prime}=\vec{r}_{i}+\delta \vec{r}$.

(4) If the moved particle has an overlap with

- no other particle, accept the step.

- one particle, go back to operation 3 and displace the new particle (i.e. the particle which has the overlap with the old one) by $\delta \vec{r}$.

- two or more particles, reject the step and start from the beginning.

An illustration of this process is given in figure 1. Obviously, this updating process is ergodic and satisfies the detailed balance condition. The algorithm

2 A lattice version of this algorithm is given in [12].

3 The region and the distribution of the new position could be of other types. For simplicity, we just consider the case in the text. 
fig_schema.eps

Fig. 1. Schematic illustration of an improved Metropolis step for a hard disk system. Light grey disks indicate the initial positions, while dark grey disks show the final ones. The process started with disk ' 1 ' and ended at ' 4 '.

is constructed in such a way that it produces long chains in ordered areas (i.e. when the position correlation length is large), while disordered regions lead to short chains. Consequently the new algorithm spends more time in the ordered domains. The stability of these ordered regions is responsible for the large autocorrelation in the two-dimensional hard disk system, so that the new non-local algorithm can lead to a decrease in the autocorrelation times.

Moreover, the updating process can be modified in such a way that the maximum number of moved particles is restricted to $k_{\max }$, i.e. the step is rejected if the number of particles in the chain exceeds $k_{\max }$. The value of the step size $\epsilon$ for the improved Metropolis algorithm as well as for the original algorithm have to be tuned in such a way that autocorrelation times are minimized.

\section{Autocorrelation times}

In order to test the improvement of the chain Metropolis algorithm, we studied the two-dimensional hard disk system in the $N V T$ ensemble in the vicinity of the transition density $\rho_{\mathrm{c}} \approx 0.898$ [6], where $\rho$ is given reduced units. We used a square box with periodic boundary conditions and measured the normalized autocorrelation function $\Gamma_{\mathrm{O}}(t)$ for an observable $O$ which is defined as

$$
\Gamma_{\mathrm{O}}(t)=\frac{\langle O(0) O(t)\rangle-\langle O\rangle^{2}}{\left\langle O^{2}\right\rangle-\langle O\rangle^{2}},
$$

where $t$ indicates the fictitious Monte Carlo time. As an example, figure 2 shows the autocorrelation function for the fractional number of defects

$$
n_{\mathrm{def}}=\sum_{i \neq 6} N_{i} / N
$$






Fig. 2. Autocorrelation functions for the fractional number of defects. The hard disk system contained 1024 particles at $\rho=0.870$. 'M' and ' $\mathrm{C}$ ' denote the original and the chain Metropolis updating scheme, respectively. The step sizes are given in units of the step size for the Metropolis algorithm $\epsilon^{\mathrm{M}} \cdot \epsilon^{\mathrm{M}}$ was chosen in such a way that the acceptance rate was about $50 \%$, which yields approximately the optimal value, i.e. minimal autocorrelation times (slight deviations in $\epsilon^{\mathrm{M}}$ from the optimal value lead only to non-essential changes).

where $N$ is the total number of particles and $N_{i}$ the number particles with $i$ neighbours determined by the Voronoi construction [14]. The observable $n_{\text {def }}$ characterizes the defect structure of the two-dimensional system. For the conventional Metropolis algorithm, particles were selected sequentially in order to cut down the amount of random number generation. A sweep corresponds to one trial of changing the coordinates for every particle. For the chain Metropolis algorithm, particles have to be chosen randomly. In this case, we define a 'sweep' as $N$ different trials to move chains of particles. Obviously, for the same step size $\epsilon\left(\epsilon^{\mathrm{C}} / \epsilon^{\mathrm{M}}=1.0\right)$, the improved update scheme leads to a faster decrease of the autocorrelation function. This can be easily explained by the fact that every displacement of a particle $i$ accepted by the conventional Metropolis step will also be accepted by the improved step. An increase of the step size $\epsilon$ for the chain Metropolis algorithm leads to a faster decrease of the autocorrelation function. However, for large values of $\epsilon$ a further increase results in an increase of the autocorrelation function.

A realistic comparison of the new and the conventional Metropolis algorithm has to be done with autocorrelation times measured in computer time, since a step of a chain of particles is more time-consuming. Therefore, we calculated 
the integrated autocorrelation time

$$
\tau_{\mathrm{int}, \mathrm{O}}=\frac{1}{2}+\sum_{t=1}^{\infty} \Gamma_{\mathrm{O}}(t)
$$

in sweeps and in CPU time. Again, the values for the observable $n_{\text {def }}$ are collected in table 1 . The new algorithm leads to a substantial decrease in the integrated autocorrelation time. The improvement is better for large $k_{\max }$. For the largest step size $\epsilon^{\mathrm{C}}$, the autocorrelation time measured in CPU time seems to increase. It is not clear if this increase already sets in or if it is just a statistical effect. However, the exact minimum of $\tau(\epsilon)$ is not very important, since it is flat.

In addition to the fractional number of defects, we studied the second moment of the bond orientational order parameter

$$
\psi_{6}^{2}=\left\langle\left|\frac{1}{N} \sum_{j} \frac{1}{N_{i}} \sum_{k=1}^{N_{i}} \exp \left(6 \mathrm{i} \theta_{k j}\right)\right|^{2}\right\rangle,
$$

where the first sum is over all particles, the sum on $k$ is over the $N_{i}$ neighbours of the particle $j$, and $\theta_{k j}$ is the angle between the particles $k$ and $j$ and an arbitrary but fixed reference axis. The bond orientational order parameter is, in contrast to the defect density, a global quantity and describes the orientational order. The values of $\psi_{6}{ }^{2}$ lie between 0 and 1 , where the latter corresponds to a perfect crystalline structure.

Integrated autocorrelation times for the second moment of the bond orientational order parameter are larger. For example, for the 4096 particle system at $\rho=0.870, \tau_{\psi_{6}{ }^{2}}^{\mathrm{C}} / \tau_{n_{\mathrm{def}}}^{\mathrm{C}} \approx 3.5$. Indeed, the stability of large crystalline regions prevents large changes of the global quantity $\psi_{6}{ }^{2}$. Therefore, the determination is more time-consuming, and more attention was given to the defect density. Furthermore, the investigations of $\psi_{6}{ }^{2}$ point to similar conclusions as those of $n_{\text {def }}$. For instance at $\rho=0.870$ and $\epsilon^{\mathrm{C}} / \epsilon^{\mathrm{M}}=1.55, \tau^{\mathrm{C}} / \tau^{\mathrm{M}}=0.15$ (in computer time) for 1024 particles, and $\tau^{\mathrm{C}} / \tau^{\mathrm{M}}=0.32$ for 4096 particles were obtained for $\psi_{6}{ }^{2}$. Some runs at $\rho=0.9$ and $\epsilon^{\mathrm{C}} / \epsilon^{\mathrm{M}}=2.0$ with 4096 and 16384 hard disks were also performed, yielding an estimate of $\tau^{\mathrm{C}} / \tau^{\mathrm{M}} \approx 0.2$.

Investigations of the computer time used showed that it approximately scales with the number of particles (for systems with more than 1024 particles) for both densities. This is the expected behaviour for the local Metropolis algorithm. In the case of the improved algorithm it indicates that large chains play no significant role. We checked this by studying the chain length in systems with 4096 particles at both densities. Indeed, chains with more than 16 particles are very rare. Therefore, one might expect that the chain Metropolis 
Table 1

Integrated autocorrelation times of the improved Metropolis algorithm for the fractional number of defects $n_{\text {def }}$. The acceptance rate for the conventional Metropolis algorithm was 53-55\%, which corresponds approximately to the optimal value of $\epsilon^{\mathrm{M}}$ (which minimizes the integrated autocorrelation time in sweeps as well as in CPU time). In this case the particles were selected sequentially to cut down the amount of random number generation.

\begin{tabular}{ccccccc}
\hline \hline$\rho$ & $N$ & $\epsilon^{\mathrm{C}} / \epsilon^{\mathrm{M}}$ & $k_{\max }$ & $\begin{array}{c}\text { acceptance } \\
\text { rate }\end{array}$ & $\begin{array}{c}\tau^{\mathrm{C}} / \tau^{\mathrm{M}} \\
{[\text { sweeps] }}\end{array}$ & $\begin{array}{c}\tau^{\mathrm{C}} / \tau^{\mathrm{M}} \\
{[\text { CPU time] }}\end{array}$ \\
\hline 0.870 & 1024 & 1.00 & $N$ & $88 \%$ & 0.24 & 0.34 \\
0.870 & 1024 & 1.27 & $N$ & $81 \%$ & 0.13 & 0.20 \\
0.870 & 1024 & 1.27 & 6 & $80 \%$ & 0.14 & 0.22 \\
0.870 & 1024 & 1.27 & 3 & $74 \%$ & 0.23 & 0.32 \\
0.870 & 1024 & 1.55 & $N$ & $73 \%$ & 0.11 & 0.18 \\
0.870 & 1024 & 1.91 & $N$ & $63 \%$ & 0.069 & 0.11 \\
0.870 & 4096 & 1.91 & $N$ & $63 \%$ & 0.14 & 0.31 \\
0.870 & 1024 & 2.27 & $N$ & $53 \%$ & 0.070 & 0.12 \\
\hline 0.898 & 1024 & 1.00 & $N$ & $86 \%$ & 0.18 & 0.40 \\
0.898 & 1024 & 1.27 & $N$ & $79 \%$ & 0.12 & 0.29 \\
0.898 & 1024 & 1.27 & 6 & $78 \%$ & 0.14 & 0.34 \\
0.898 & 1024 & 1.27 & 3 & $72 \%$ & 0.24 & 0.53 \\
0.898 & 1024 & 1.55 & $N$ & $70 \%$ & 0.11 & 0.27 \\
0.898 & 4096 & 1.55 & $N$ & $70 \%$ & 0.14 & 0.32 \\
0.898 & 1024 & 1.91 & $N$ & $59 \%$ & 0.12 & 0.29 \\
\hline \hline
\end{tabular}

algorithm does not lead to an improvement of the dynamical critical exponent. Integrated autocorrelation times for the 4096 particle system at the critical density seem to confirm this assumption, but are affected by large statistical errors. Hence it appears that critical slowing down is not reduced.

\section{Modification}

A further improvement can perhaps be achieved by an updating scheme which enables the interaction of particles over large distances. Since the algorithm described above stops if more than two particles overlap, this situation must 
be avoided. Therefore, each particle is moved in a chosen direction just to the point where it collides with another particle. For simplicity, we consider the case where all particles are moved in the same direction $\vec{v}$. With the number of particles $k$ in the chain chosen arbitrarily, this translates into:

(1) Select randomly a number $k$ (the number of particles to move) between 1 and $k_{\max }$. Select randomly with equal probability a particle $i$ (the first particle in the chain) from the whole system.

(2) Choose a vector $\vec{v}$ of the surface of a unit sphere, i.e. with a probability $\sim d \Omega$.

(3) Move the particle into the direction of $\vec{v}$ until a collision with another particle occurs. Add this to the chain.

(4) If the number of particles in the chain (including the new one) is

- lower than $k$, go back to operation 3 and move the new particle in the same direction.

- equal to $k$, place the particle with uniform probability between its initial position and the collision point (in $\vec{v}$ direction), i.e. $\vec{r}_{k} \rightarrow \vec{r}_{k}{ }^{\prime}=\vec{r}_{k}+$ $r\left(\vec{r}_{k}^{\text {coll }}-\vec{r}_{k}\right)$, where $r$ denotes a uniform distributed random number between 0 and 1 .

(5) Accept the new configuration (the displacement of the whole chain) with probability $\min \left(1, l_{\text {end }} / l_{\text {start }}\right) . l_{\text {end }}=\left|\vec{r}_{k}^{\text {coll }}-\vec{r}_{k}\right|$ is the length between the initial position and the collision point of the last particle; $l_{\text {start }}$ is the length from the final position of the first particle to the collision point in $-\vec{v}$ direction.

This updating step and the meaning of $l_{\text {start }}$ is explained schematically in figure 3. The new accept/reject step (operation 5) is necessary to fulfill the detailed balance condition. The case $k=1$ is realized by placing the particle randomly between the collision points in positive and negative $\vec{v}$ direction. This step is always accepted. The value of $k_{\max }$ as well as the distribution of $k$ between 1 and $k_{\max }$ (which is not necessarily chosen with equal probability) have to be tuned in such a way that autocorrelation times are minimal.

The advantage of this updating scheme is a high and length-independent probability to accept a step, which perhaps can counter critical slowing down. On the other hand, a displacement of a chain with $k$ particles is more timeconsuming due to the additional calculations of the collision points than a comparable step in the first (improved) updating scheme. Therefore, a detailed investigation of this algorithm, in particular for the dynamical critical exponent, is necessary in order to test the performance. So far only a few runs have been performed to ensure that it yields the correct expectation values and to get a rough estimate of the integrated autocorrelation time for $\psi_{6}{ }^{2}$. For 16384 hard disks at $\rho=0.9$, the ratio of the integrated autocorrelation time (in sweeps) of this updating scheme to those of the first scheme, $\tau^{\mathrm{C}^{\prime}} / \tau^{\mathrm{C}}$, was about 0.22 , while the value measured in CPU time strongly depends on the 


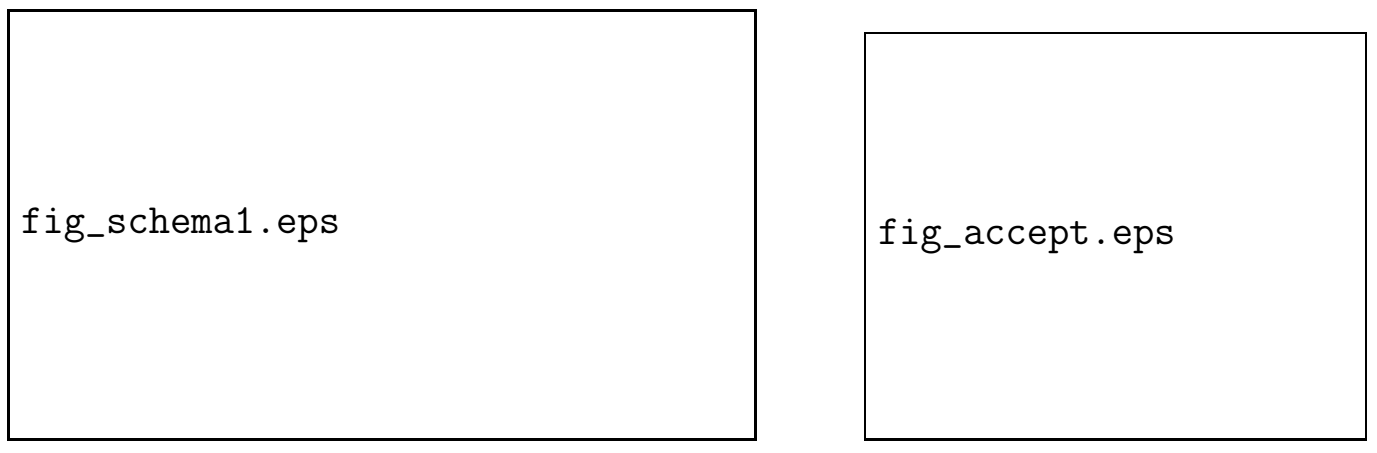

Fig. 3. The left figure illustrates the second updating scheme. A particle (' 1 ') is moved in a randomly chosen direction until it collides with another one ('2'). The second particle is moved into the same direction up to the next collision. This process is stopped after $k$ particles. The last particle is randomly placed between its initial position and the collision point in $\vec{v}$ direction. The right figure illustrates the length $l_{\text {start }}$, which is marked by an arrow. The disk in the middle is the initial position of particle ' 1 ', the right disk shows the final position, while the left one corresponds to the collision point in $-\vec{v}$ direction. The length $l_{\text {start }}$ is now the distance between the final position and the collision point in the negative $\vec{v}$ direction.

implementation of the algorithms.

\section{Conclusions}

In summary, we presented a non-local Metropolis algorithm for arbitrary hard core systems in any dimension. We investigated the two-dimensional hard disk system for a detailed comparison between the conventional local and improved non-local algorithm. The Monte Carlo simulations showed that the new algorithm leads to an essential reduction of integrated autocorrelation times for the fractional number of defects as well as the global bond orientational parameter. An additional modification which perhaps leads to a further improvement was presented, but no investigations were made.

It is hoped that this algorithm will help to resolve the type and order of the phase transition in the two-dimensional hard disk system. Work along this line is in progress.

\section{Acknowledgements}

Critical comments by Joachim Hein, Karl Jansen and Jacques Mainville are gratefully acknowledged. We thank Harro Hahn for useful discussions. 


\section{References}

[1] K.J. Strandburg, Rev. Mod. Phys. 60 (1988) 161.

[2] J.M. Kosterlitz, D.J. Thouless, J. Phys. C 6 (1973) 1181;

J.M. Kosterlitz, J. Phys. C 7 (1974) 1046;

B.I. Halperin, D.R. Nelson, Phys. Rev. Lett. 41 (1978) 121;

A.P. Young, Phys. Rev. B 19 (1979) 1855.

[3] S.T. Chui, Phys. Rev. Lett. 48 (1982) 933; Phys. Rev. B 28 (1983) 178.

[4] B.J. Alder, T.E. Wainwright, Phys. Rev. 127 (1962) 359.

[5] J.A. Zollweg, G.V. Chester, Phys. Rev. B 46 (1992) 11186.

[6] H. Weber, D. Marx, K. Binder, Phys. Rev. B 51 (1995) 14636.

[7] J. Lee, K.J. Strandburg, Phys. Rev. B 46 (1992) 11190.

[8] J.F. Fernández, J.J. Alonso, J. Stankiewicz, Phys. Rev. Lett. 75 (1995) 3477.

[9] R.H. Swendsen, J.S. Wang, Phys. Rev. Lett. 58 (1987) 86.

[10] U. Wolff, Phys. Rev. Lett. 62 (1989) 361.

[11] C. Dress, W. Krauth, J. Phys. A: Math. Gen. 28 (1995) L597.

[12] J.R. Heringa, H.W.J. Blöte, Physica A 232 (1996) 369.

[13] N. Metropolis, A.W. Rosenbluth, M.N. Rosenbluth, A.H. Teller, E. Teller, J. Chem. Phys. 21 (1953) 1087.

[14] For a definition of Voronoi cell see: D.P. Fraser, M.J. Zuckerman, O.G. Mouritzen, Phys. Rev. A 42 (1990) 3186. 




